\title{
Empirical Investigation of Instant Messaging Security in a Virtual Environment
}

\author{
Peter S. Nyakomitta \\ School of informatics and \\ Innovative systems \\ Jaramogi Oginga Odinga \\ University of Science and \\ Technology \\ Kenya
}

\author{
Dr. Solomon Ogara \\ School of informatics and \\ Innovative systems \\ Jaramogi Oginga Odinga \\ University of Science and \\ Technology \\ Kenya
}

\author{
Dr. Silvance Abeka \\ School of informatics and \\ Innovative systems \\ Jaramogi Oginga Odinga \\ University of Science and \\ Technology \\ Kenya
}

\begin{abstract}
Use of instant messaging services is becoming increasingly popular with Internet based systems like America Online's Instant Messaging (AIM), Microsoft's MSN Messenger, Yahoo! Messenger, WhatsApp, Viber, Kakaotalk, Skype and face book instant messenger. These tools support any process where quick response and rapid problem solving are needed, and where faster communication than emails or telephones is useful. More and more people are enjoying the convenience and simplicity provided by the real-time messaging systems in their day-to-day life. Moreover, the instant messaging services have also found applications in business. In this application domain, the instant messaging services are employed for communicating with customers and partners, offering customer support, receiving real-time alerts, as well as management and project coordination. Despite their heavy utilization, public instant messaging systems have been criticized for having a number of security weaknesses. These weaknesses originate from the facts that the instant messaging clients are always on, those logs can contain sensitive information, and that the communication goes through an externally controlled server. Most of the instant messaging services were never intended for secure communication in the first place. The rapid growth in the number of public instant messaging users has therefore created a new security concern for information technology managers. In this paper, a prototype instant messaging was developed and employed to investigate some of the security challenges in instant messaging applications. The results indicated that upon following the TCP stream, the instant messages were in plain text in the sending and receiving communication devices interfaces and therefore prone to eavesdropping. As such, the researchers propose a port-based algorithm that would scramble the data packets at the end devices, requiring the users to input decryption keys for the data to be transformed into human readable format.
\end{abstract}

Keywords: Instant Messaging (IM), Plain Text, prototype, Client, Server, Security

\section{INTRODUCTION}

The potential threats when using instant messaging services include the spread of malicious code, instant messaging software vulnerabilities, leakage of sensitive information, monitoring and retention issues, and lack of accountability. In their study, Weissbrot and Alison (2016) explain that the enterprise usage of instant is growing in both volume and importance. The use of these tools benefit their users in facilitating faster decision-making process, higher productivity and lower telecommunication costs. At the same time as, the instant messaging threats such as viruses are rapidly gaining attention as attackers begin to shift their focus from better-protected email systems to these networks. 
Moreover, Mark (2015) report that spam messages can also be spread through the instant messaging tools. The spam that a user receives via these services is referred to as spim. Popular instant messaging clients have, just like any other software application, have a history of common security vulnerabilities. This means that installing an instant messaging client has the potential of introducing new vulnerabilities to a computer system. Confidentiality, which deals with the protection of organizational data or government data from illegal access, is a major concern when using a public instant messaging service for communication. This so according to Vinnie and Belvin, (2012), because in public instant messaging networks, communications exchanged between users are normally routed through instant messaging server farms which are controlled by the service providers themselves. In situations where client instant messaging software has a peerto-peer capability, users can communicate with each other without passing through these servers.

Lin et al., (2016) note that no matter which mode is being utilized for communication purposes, this traffic is vulnerable to eavesdropping because most public clients do not possess any encryption capability. The consequences are that it is possible for sensitive information to be read or sniffed by unauthorized users. The situation can be even worse when public instant messaging services are used to communicate with individuals outside an organization. This may lead to the leakage of sensitive organizational classified data.

\section{LITERATURE REVIEW}

In this section, the researcher discuss on the literature concerning the related work to the research, which is empirical investigation of instant messaging security in a virtual environment.

\subsection{RELATED WORK}

In his research work, Wendell (2013) found out that the protocols employed by public instant messaging services are often considered rogue protocols. This because they were specifically designed to evade standard security controls. The consequences are that not only can instant messaging clients be configured to connect through SOCKS or web proxy servers, but as Green (2014) point out, the protocols are also capable of finding their way out through the firewall on their own. They can do this by determining an open port such as transmission control protocol (TCP) port 80 , or by tunneling their traffic inside the hypertext transfer protocol (HTTP) requests. These practices make these traffic unrecognizable from standard web traffic. Additionally, the scripting and file transfer capabilities of instant messaging systems might expose an organization to leaks of sensitive information.

According to Andreas and Buchenscheit (2014), most of the productive features provided by the instant messaging applications are only one side of the coin. This is because these applications have vulnerabilities that related to the underlying instant messaging technology. Consequently, these vulnerabilities expose user communications to a number of security threats. Therefore, communication via these applications is regarded insecure. To start with, Jagwani (2016) note that all of the messages and connection information are retained on the application providers' servers. This means that the information communicated across the network is controlled by the provider of the instant messaging utility.

Another serious challenge with instant messaging connections is that the messaging process normally happens in plain text (Frosch et al., 2016). Consequently, this renders them susceptible to eavesdropping. Additionally, instant messaging client software quite often requires the user to expose their open user 
datagram ports. This gives rise to the threat posed by potential security vulnerabilities of user datagram protocol (UDP) ports. As Smith (2013) points out, UDP is vulnerable to spoofing and denial of service attacks. On the other hand, it is not feasible to spoof an address across the internet using transmission control protocol (TCP). This is because the three way handshake will never complete. Moreover, there are features of instant messaging applications that are threats to security. Such features include presence and status broadcasting, interoperability with others, maintaining a lists of all desired contacts, use of third party servers to provide chat functionality to messenger clients and keeping a log of messages and other events/ activities (Fahrnberger, 2014).

\section{RESEARCH METHODOLOGY}

This section presents the way the study was carried out. It involves the various steps that the researcher adopted in studying the research problem as well the logic behind those steps which include the research prototyping approach and the procedure is provided as outlined below.

\subsection{Prototyping Approach}

In this paper, a model that could help demonstrate the security challenges in instant messaging applications in a virtualized environment was developed. The hypervisor was chosen to be Oracle VM Virtual box. This hypervisor is a cross-platform virtualization application, meaning that it installs on the existing Intel or AMD-based computers,. It supports operating systems such as Windows, Mac, Linux or Solaris. It serves to extend the capabilities of the existing computer so that it can run multiple operating systems inside multiple virtual machines at the same time. Using this hypervisor, one can install and run as many virtual machines as he likes. The only practical limiting factors are disk space and memory. Moreover, it is very simple to use and very powerful. This is because it can run on any platform, ranging from small embedded systems or desktop class machines to datacenter deployments and even Cloud environments. This made it the best choice for this paper. Figure 1 shows the interface for VirtualBox hypervisor.

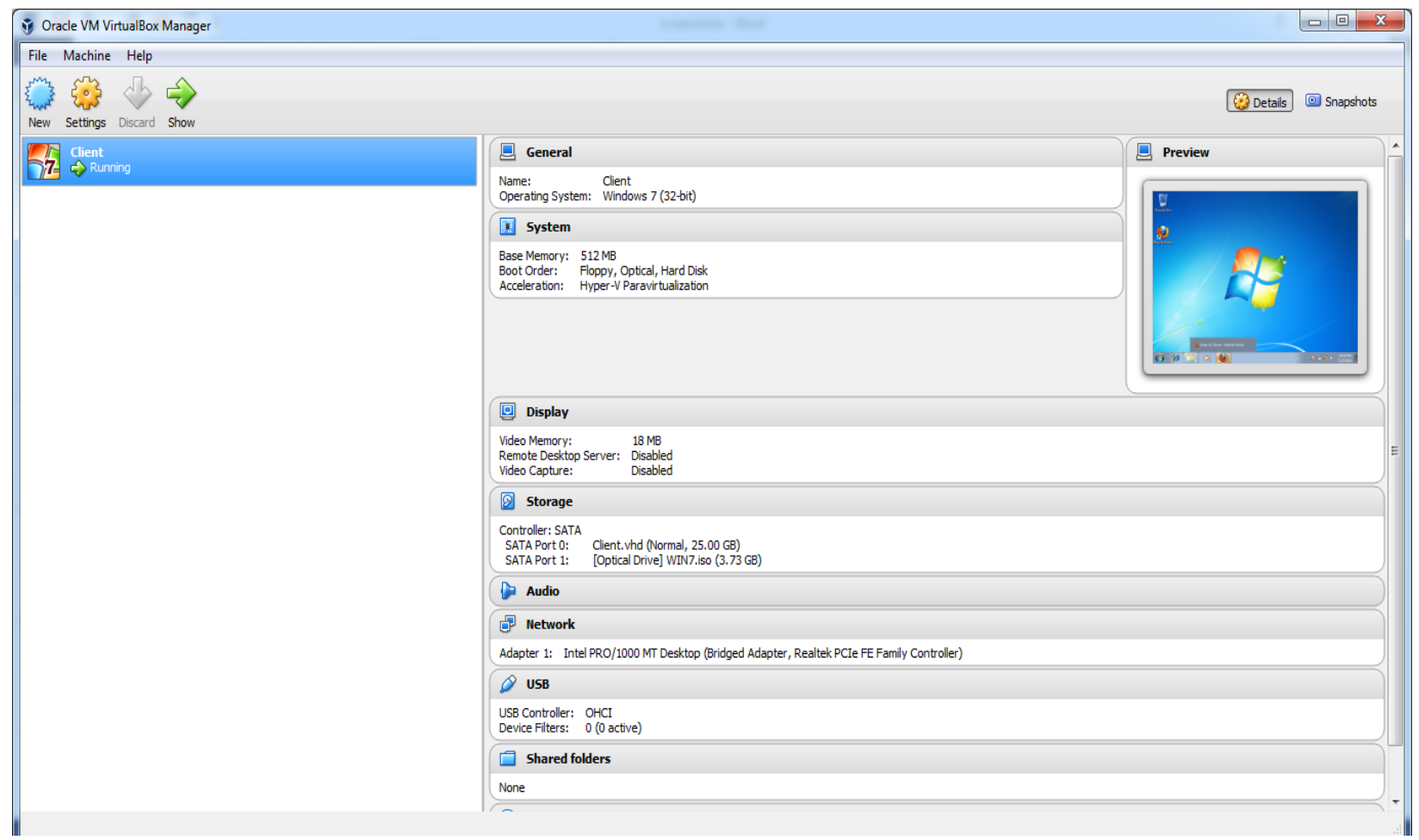

Figure 1: VirtualBox Interface 


\subsection{Procedure}

After the installation of the VirtualBox hypervisor, a client operating system was installed inside this hypervisor. This client was chosen to be Windows Professional, 32 bit.

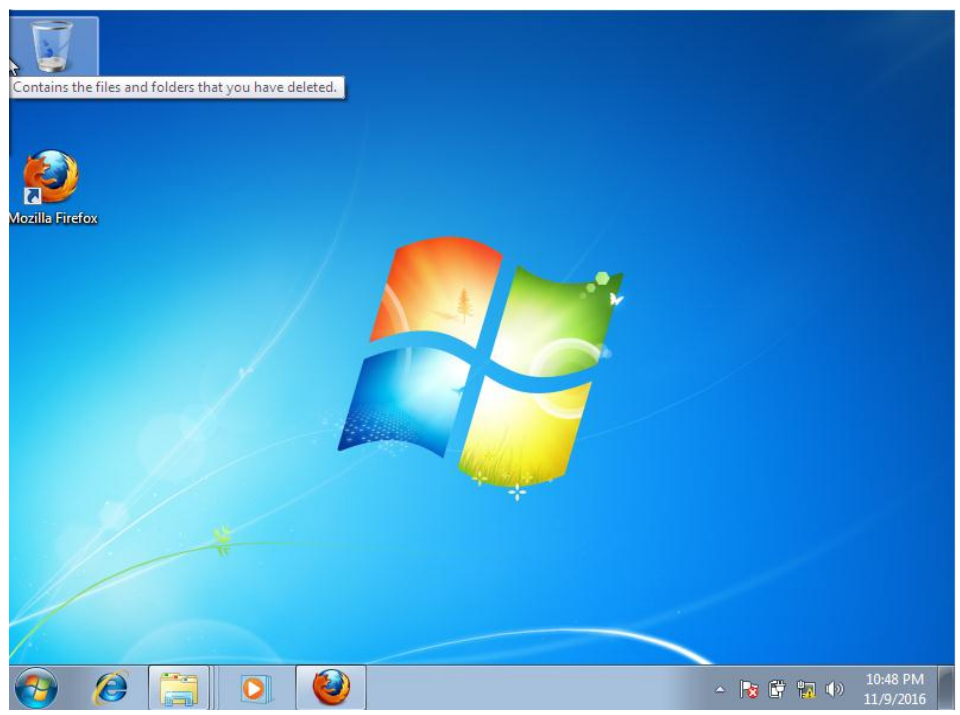

Figure 2: Client Operating System

The 32 - bit client was selected to avoid bus speed mismatch because the underlying host operating system ran 32 -bit. After this, Wamp server was installed inside this client operating system. This was meant to provide a respository for the instant messages through its PhPMyadmin feature as shown in Figure 3.

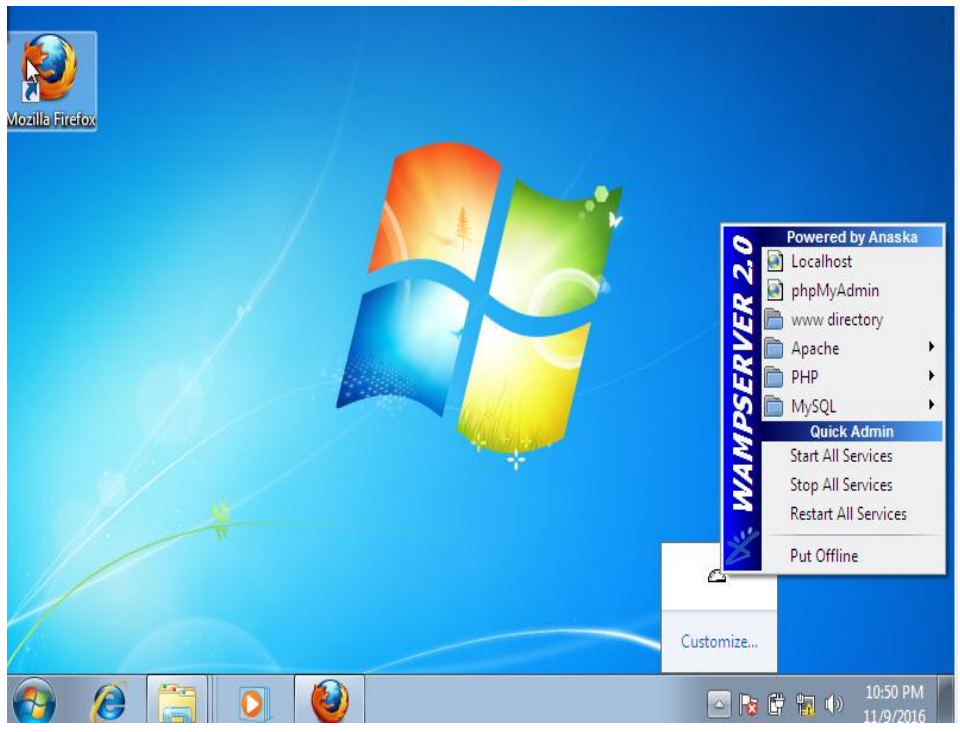

Figure 3: Wamp Server Installation 
All the $P h P$ coding was done and saved in the $w w w$ folder of wamp server. The same wamp server was also installed on another machine that was required to achieve a two way instant messaging exchange. An Ethernet cable was then connected between the machines that required exchanging the instant messages and appropriate internet protocol (IP) addresses were assigned using class $\mathrm{C}$ subnet. Figure 4 show the typical set up that was employed.
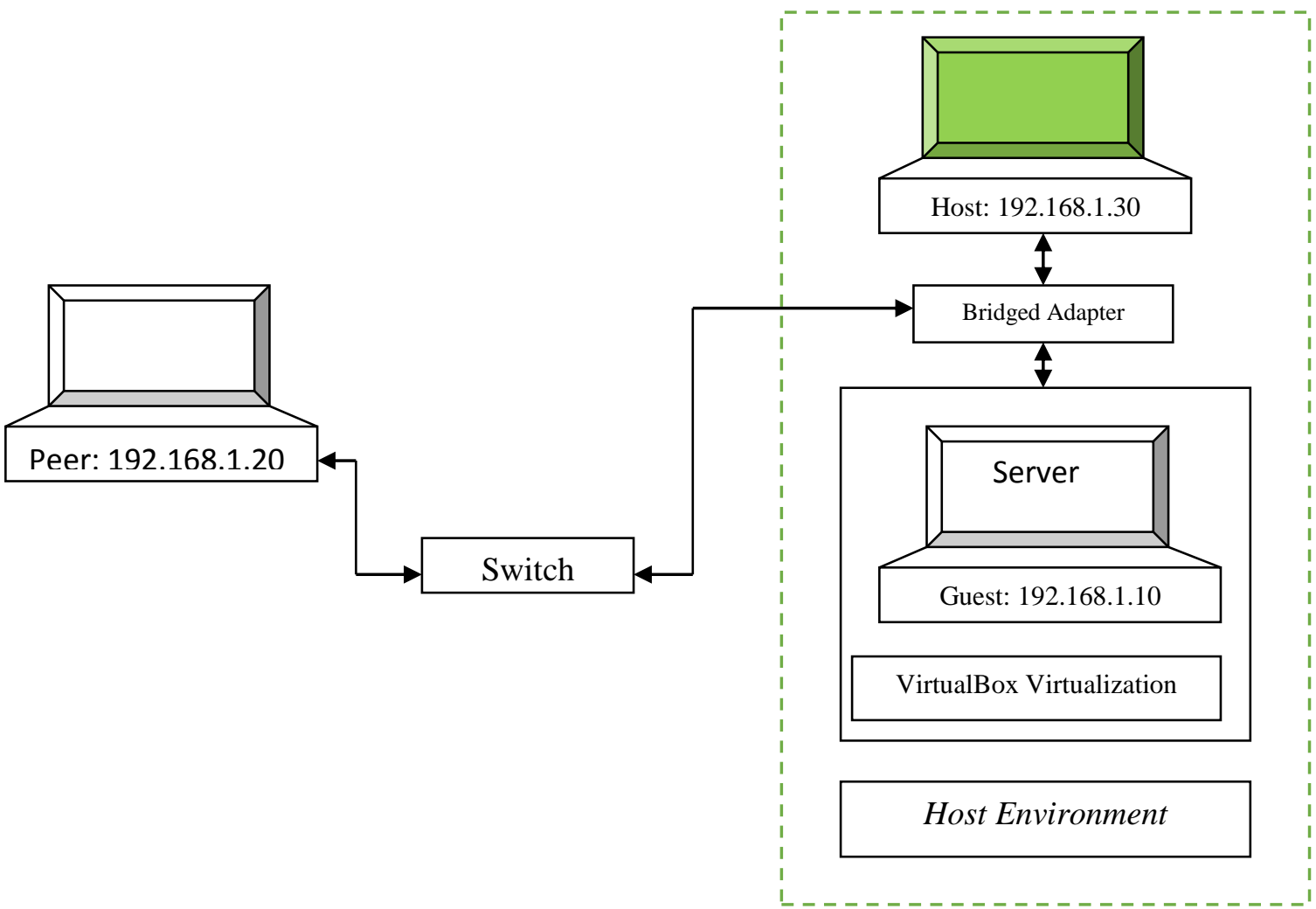

Figure 4: Prototyping Setup

In this paper, the Host operating system refers to the operating system of the physical computer on which VirtualBox was installed. On the other hand, the guest operating system was the operating system that is running inside the virtual machine. Virtual machine (VM) refers to the special environment that VirtualBox creates for the guest operating system while it is running. In other words, the guest operating system is run in a virtual machine. Basically, a virtual machine could be shown as a window on the computer's desktop, but depending on which of the various frontends of VirtualBox is in use, it can be displayed in full screen mode or remotely on another computer.

Technically, VirtualBox regards a virtual machine as a set of parameters that determine its behavior. They include hardware settings (how much memory the virtual machine should have, what hard disks VirtualBox should virtualize through which container files, or what CDs are mounted) as well as state information (whether the virtual machine is currently running, saved, or its snapshots ).

Figure 4 shows that the hypervisor was hosted in address 192.168.1.30. This hypervisor in turn hosted a client of IP address 192.168.1.10. In order for the guest operating system to communicate with the host operating system, a bridged adapter was employed as shown in Figure 4. In bridged networking, VirtualBox utilizes a device driver on the host system that filters data from the physical network adapter. 
For this reason, this driver is called a net filter driver.

This permits VirtualBox to capture data from the physical network and inject data into it. The effect of this is the creation of a new network interface in software. Ideally, when the guest operating system is utilizing such a new software interface, it looks to the host system as though the guest were physically connected to the interface using a network cable. Effectively, the host can send data to the guest through that interface and receive data from it. This means that one can set up routing or bridging between the guest and the rest of your network.

To enable bridged networking, the Settings dialog of a virtual machine was opened; from there navigation was done to the Network page. Finally, the selection of Bridged network was accomplished in the drop down list for the Attached to field as shown in Figure 5.

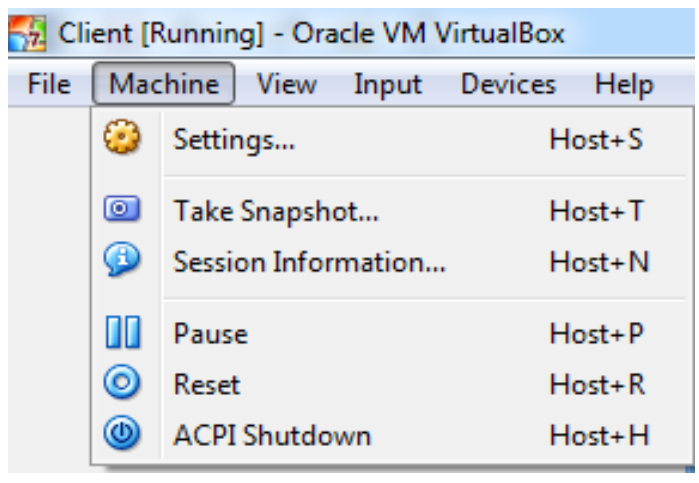

Figure 5: VirtualBox Network Setting

To finish the configuration, the desired host interface was selected from the list at the bottom of the page, which contains the physical network interfaces of the systems. As Figure 6 demonstrates, two network interfaces were detected: RealTek PCIe FE Family Controller and Qualcom Atheros QCA9565802.11b/n Wifi Adapter. Howver, since the latter adapter was for the wireless connections, the former interface RealTek PCIe FE Family Controller was selected.

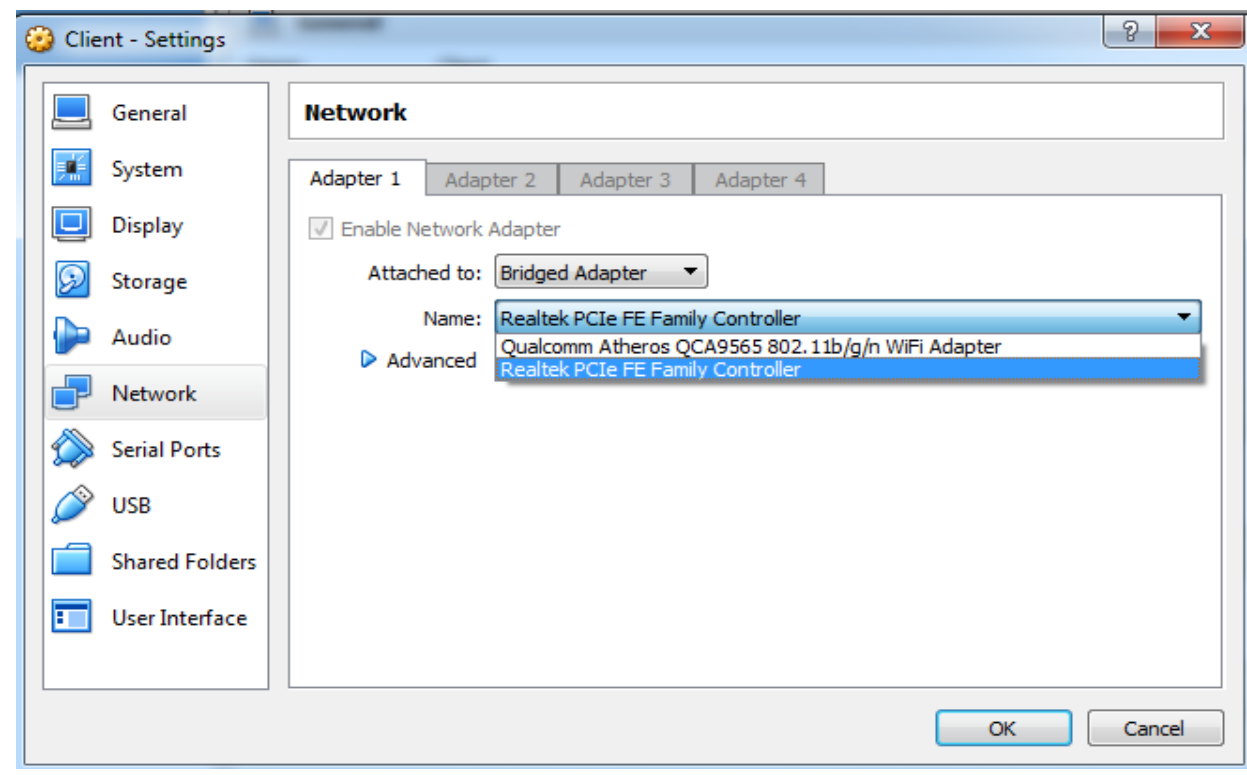

Figure 6: Bridged Host-Client Connections 
To test the effectiveness of these configurations, the internet control message protocol (ICMP) code number 8 , called packet internet groper
(PING) utility was employed. Figure 7 shows the results of the PING utility against IP address of the guest running in Oracle VirtualBox VM.

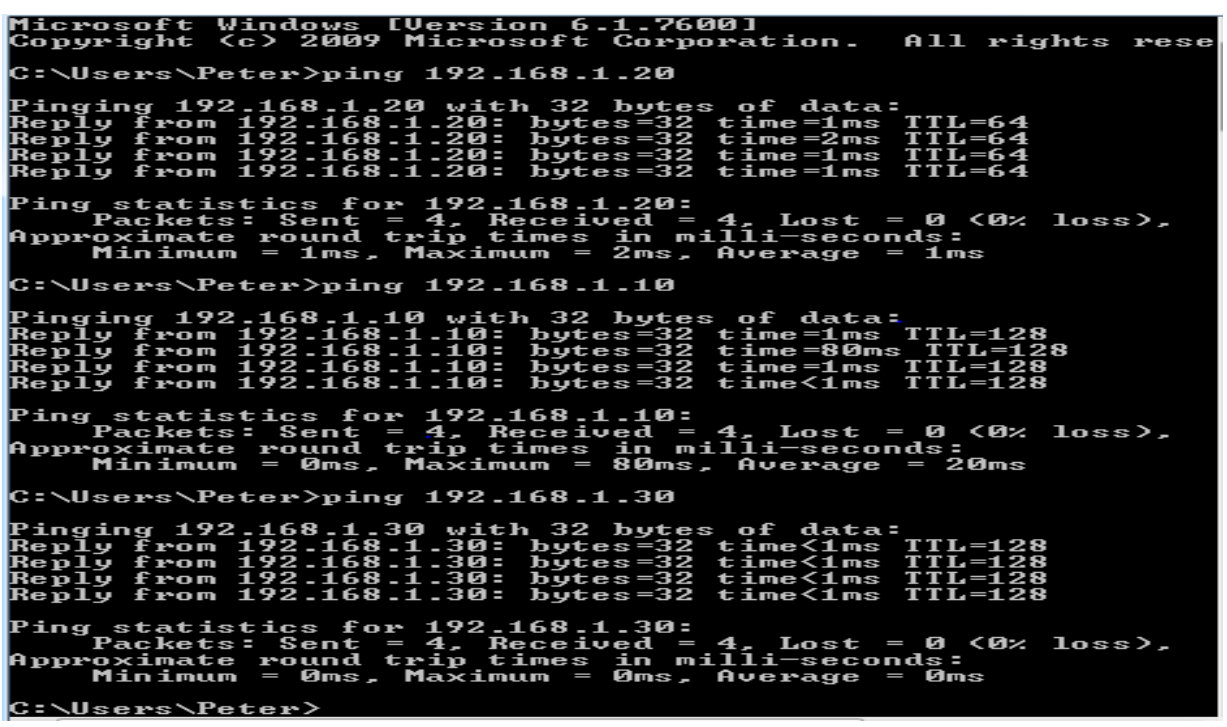

Figure 7: Connectivity Testing

As this figure shows, there was successful communication from the peer instant messaging machine to the virtualized instant messaging guest peer. This meant that all was set for the

\section{V. RESULTS AND DISCUSSION}

This section presents and discusses the results from the empirical investigation of Instant Messaging security in a virtualized environment as explained below.

\subsection{Client-Server Interface}

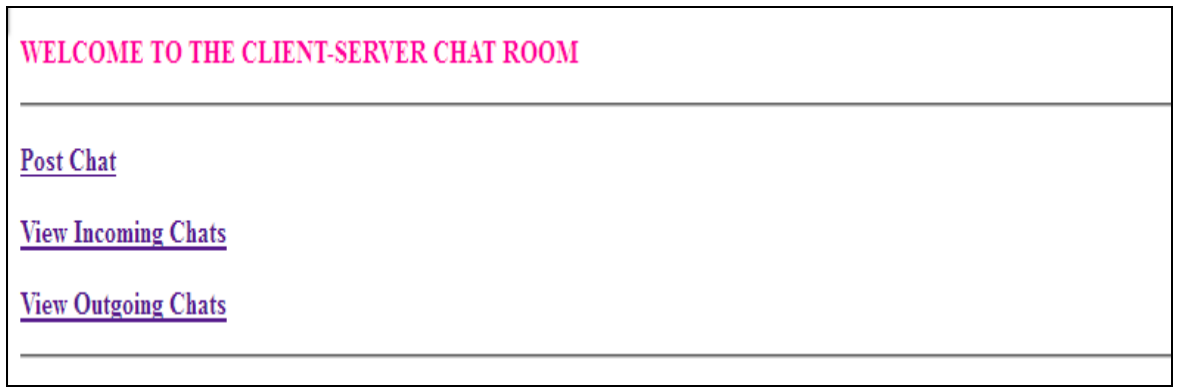

actual instant message communication between the virtualized guest and the non-virtualized peer.

Each of the instant message communicating entity had the hyperlinks shown in Figure 8. This consisted of the post Chat, View Incoming Chat and View Outgoing Chat hyperlinks. The Post Chat link was utilized to send an instant message to the receiver while the View Incoming Chat was employed to retrieve the instant message directed towards the user. Finally, the View Outgoing Chat link was used to retrieve the instant messages that the user has sent.

Figure 8: Main Chat Interface 
To post a chat, one clicks the 'Post Chat' link that then load the interface shown in Figure 9. On this interface, the user authenticates himself

\subsection{Authentication Interface}

The design represent an interface used by the. by entering a valid user name and password. Afterwards, he clicks the ' $O K$ ' button to load the next interface.

users to authenticate themselves when they $\log$ into the system.

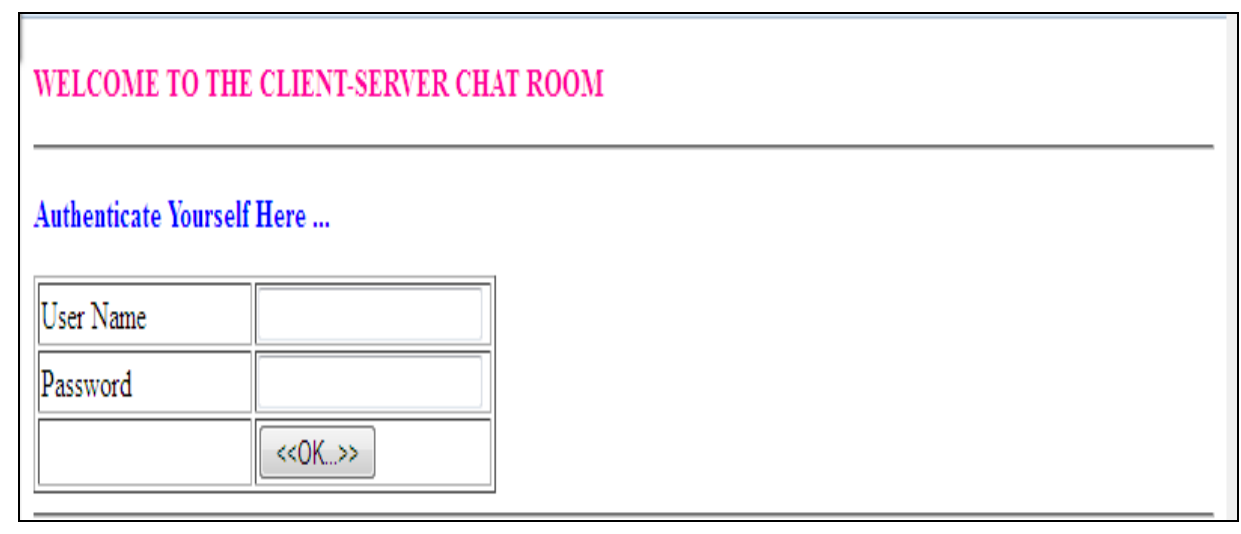

Figure 9: Authentication Interface

The username and password are analogous to the password or the unique pattern that the user draws to unlock the phone so as to access the instant messaging interface. Upon the entry of the correct credentials, the user is presented with the interface shown in Figure 10.

\subsection{Composing Interface}

In order to compose a message that will be instantly posted from the peers to the server for

processing, figure 10 represents the design.

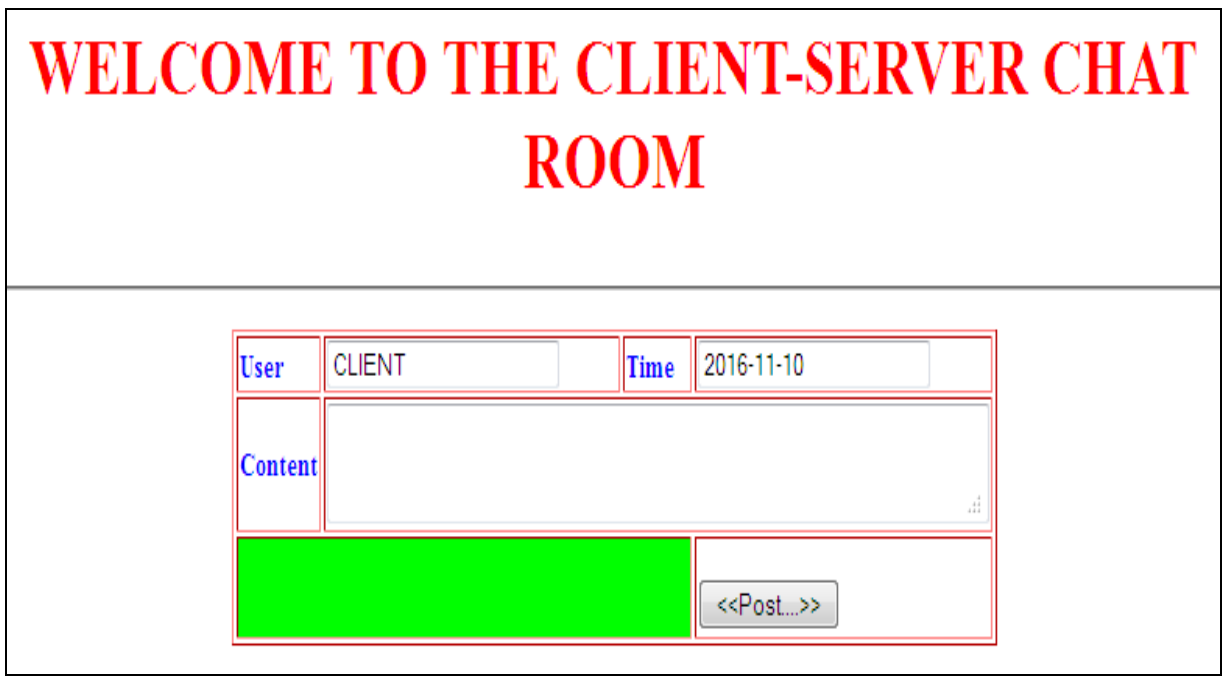

Figure 10: Chat Posting Interface 
In this interface, the user types his instant chat message and clicks the ' $O K$ ' command button.

\subsection{Posting Response}

This serves to inform the user that indeed the chat he posted has been delivered to the recipient, otherwise an error message will be
This will in turn load the interface shown in Figure 11.

displayed. To retrieve the incoming instant messages, one simply clicks on "View Incoming Chats" link of Figure 8. Upon doing this, the interface on Figure 12 will be displayed.

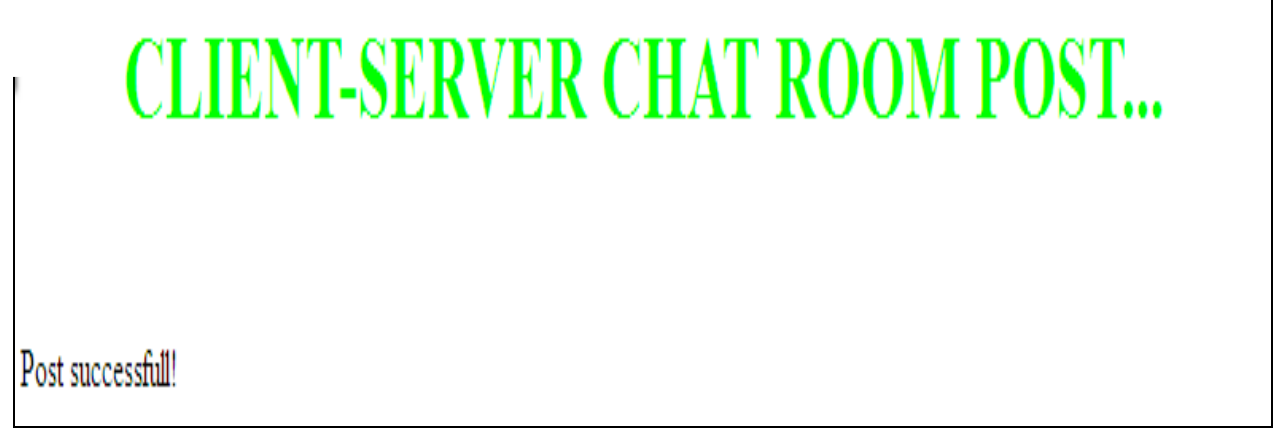

Figure 11: Instant Message Posting Successful

\subsection{Incoming Message}

This process clearly depicts what happens with

normal instant chat message retrieval applications.

\begin{tabular}{|c|c|}
\hline TSERNAIE & CHAT CONTENT \\
\hline SERVER & HI SR, BLACKOUT! \\
\hline SERVER & SO, SAD BUILDNNG COLLAPSES KILLING THREE! \\
\hline SERVER & MSC. IT SECURITY \& AUDIT IS FON.. \\
\hline
\end{tabular}

Figure 12: Incoming Chats

Typically, one clicks on the sender's contact, which then loads the chat interface from which the incoming chats can be read and post messages. To retrieve the chats sent, one clicks the "View Outgoing Chats" and this displays the information shown in Figure 13. 


\subsection{Outgoing Message}

The design demonstrate the content of the outgoing messages. On both Figure12 and Figure 13, the name of the sender and receiver are displayed on the "Username" column. The actual chat is displayed under the "Chat Content" column.

\begin{tabular}{|c|c|}
\hline USERNAME & CHAT CONTENT \\
\hline CLIENT & $\mathrm{Hi}$, Good Evening \\
\hline CLIENT & Good Afternoon \\
\hline CLIENT & How is the progress? \\
\hline CLIENT & Nightmare \\
\hline CLIENT & HI \\
\hline
\end{tabular}

Figure 13: Outgoing Chat Retrieval

Clearly, these instant message communications are in plain text, raising the susceptibility to eavesdropping. Further traffic analysis using the Wireshark software was conducted to determine whether the instant message communication can be intercepted. The results obtained are as shown in Figure 14.

\subsection{TCP Three Way Handshake communication}

The figure illustrates the synchronization process of the device during communication which describes a Three-way handshake. The TCP allows one side to establish a connection as a client and the other side to accept the connection or reject it as a serve. It shows that there was a TCP communication between the peer (IP address 192.168.1.20) and the virtualized guest (IP address 192.168.1.10).

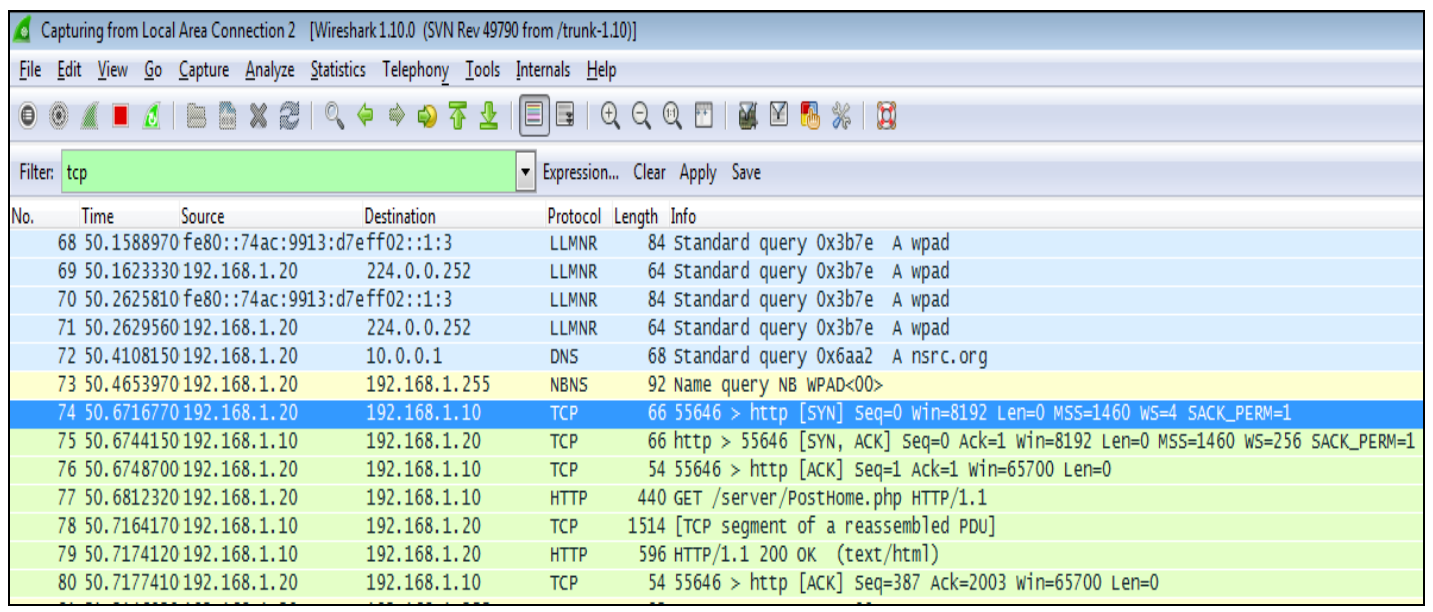

Figure 14: Instant Message Communication Interception 
The sequence number of the initial packet was 0 . The guest then respondent by acknowledging the request for connection, with $\mathrm{ACK}=1$. The peer and the virtualized guest then proceeded to exchange data using the hypertext transfer protocol (HTTP).

\subsection{Transmission Control Protocol Stream}

It's a window that details the "request" sent and the "response" received. This process was done for both the server and the client. The idea was to capture the live packet as it was being transmitted from the client to the server and
To demonstrate that the instant messages were in plain text in both sending and transmitting devices, the TCP stream was followed as shown in Figure 15.

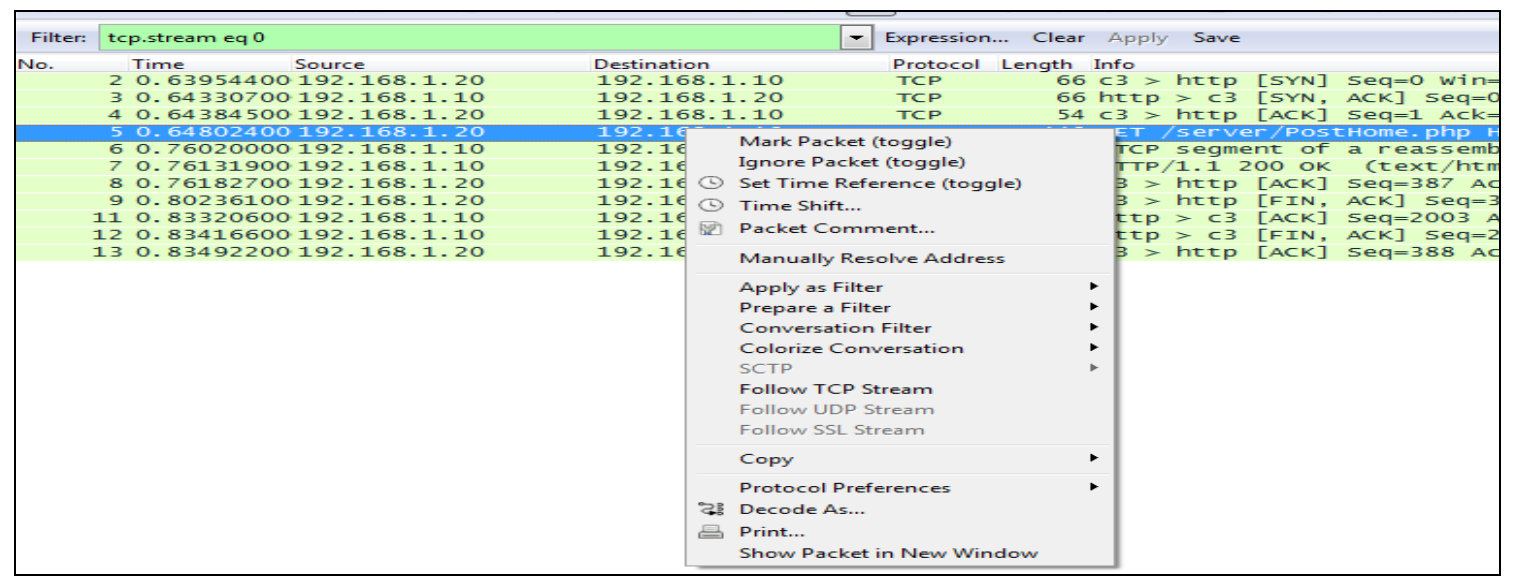

Figure 15: TCP Stream Following

\subsection{HTTP Client-Sarver Communication}

The figure depict response packet capture from from the server to the client. Since the address of the client was 192.168.1.20 and that of the server was 192.168.1.10, the following communication shown in Figure 16 was intercepted and live capture carried out.

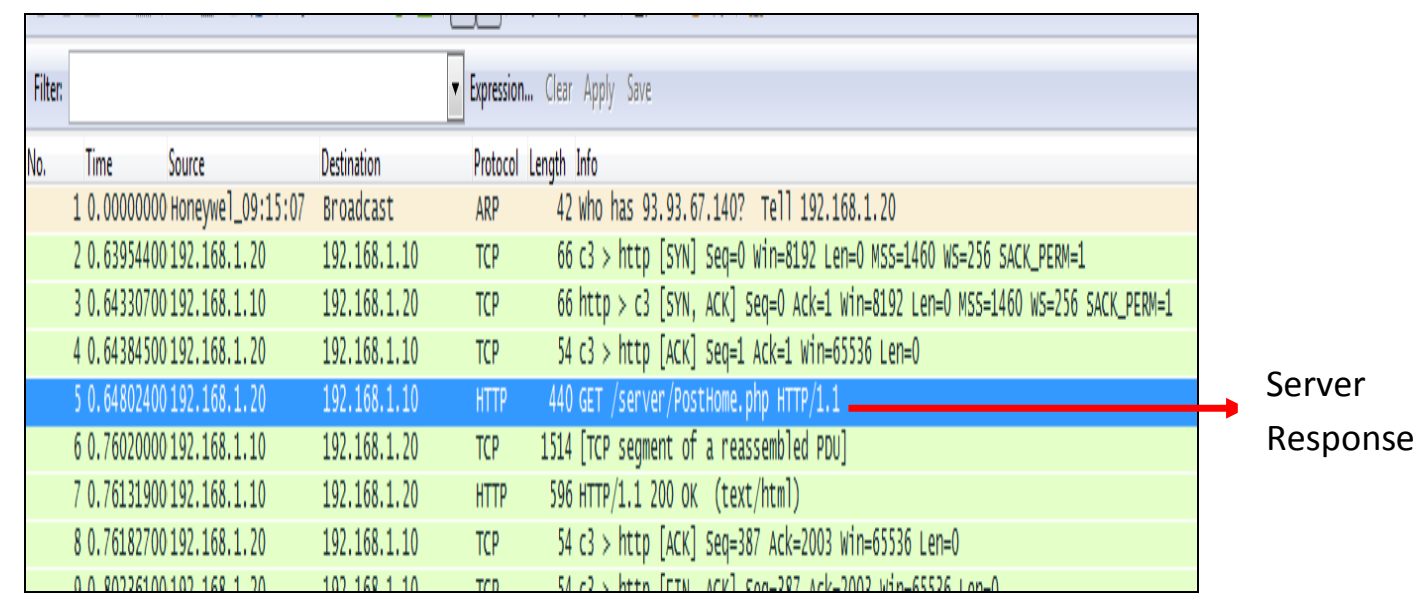

Figure 16: Client - Server Communication Interception 
Upon following this TCP stream, valuable information related to this packet was obtained

\subsection{Plaintext Client-Server Communication}

This figure shows the TCP packet in pain text. Note that Figure 17 essentially displays the contents of the table in Figure 12. To do so, it as demonstrated by Figure 17 .

uses the HTML tags for table creation $(\langle$ table $\rangle)$, table rows $(\langle t r\rangle)$, table data $(\langle t d\rangle)$.

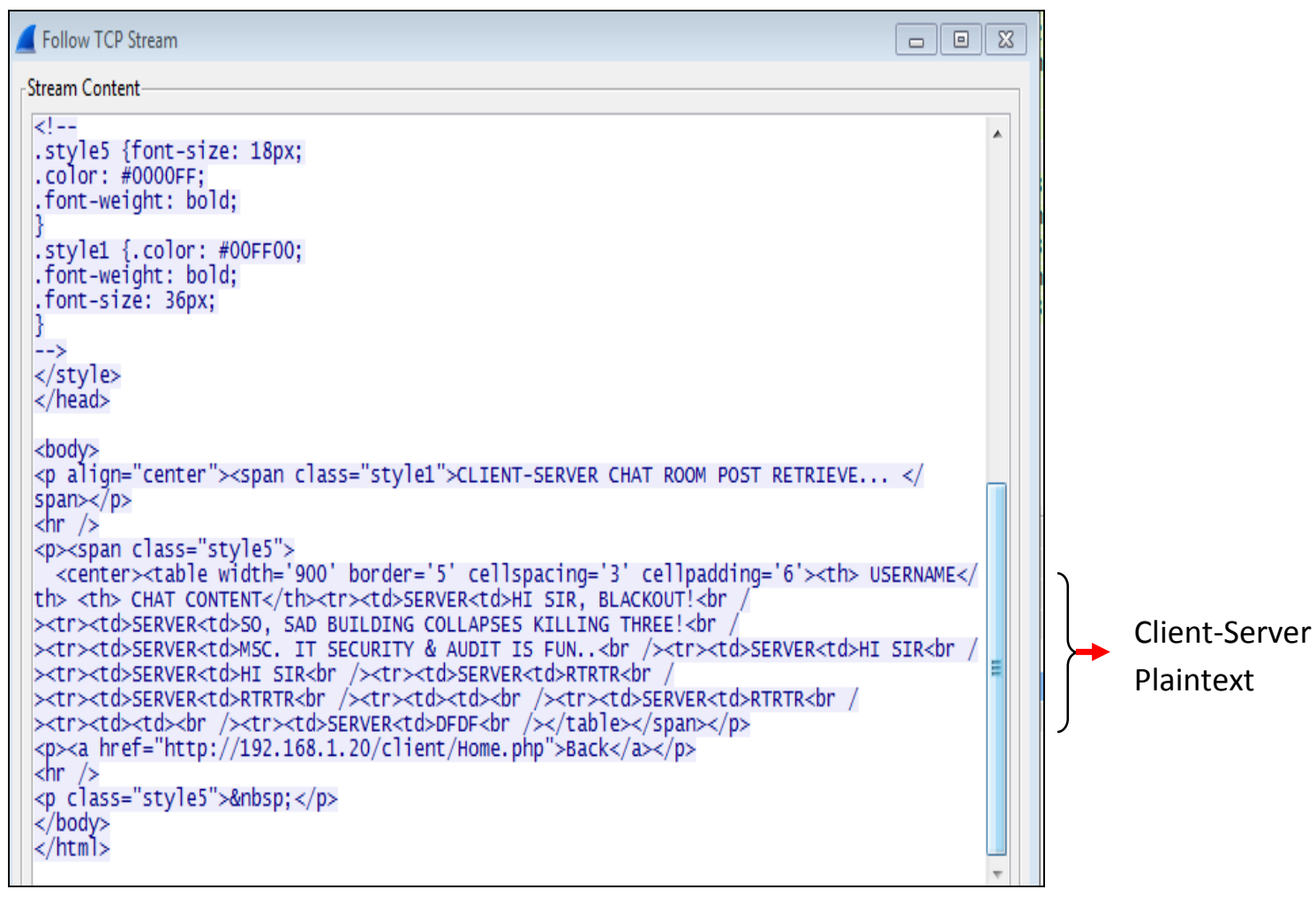

Figure 17: Plaintext Client-Server Communication

Towards the end of this TCP stream, the uniform resource locator (URL) of the machine towards which this request is directed to is given: http://192.168.1.20/client/home.php, which was the address of the client machine connecting to the server.
Obviously, this plain text packet reveals enough information that may facilitate further attacks such as spoofing attacks and denial of services (DOS) since the IP addresses and the URLs are evident from this capture. To investigate the server-client communication, the packet in Figure 18 was intercepted and followed. 


\subsection{Server - Client Communication Interception}

To demonstrate the insecure request, the analysis was done from one the peers under domain

192.168.1.20 as shown in figure 18 .

\begin{tabular}{|c|c|c|c|c|c|}
\hline \multicolumn{3}{|c|}{ Filter: } & \multicolumn{2}{|c|}{ Expression.... Clear Apply Save } & \multirow{18}{*}{$\begin{array}{l}\text { Peer } \\
\text { Packets }\end{array}$} \\
\hline \multirow[t]{17}{*}{ No. } & Source & Destination & Protoco & length Info & \\
\hline & 10.00000000 Honeywel_09:15:07 & Broadcast & & 42 who has 93.93 .67 .140 ? Tell 192.168 .1 .20 & \\
\hline & 20.63954400192 .168 .1 .20 & 192.168 .1 .10 & & $66 \mathrm{c} 3>$ http [SYN] Seq $=0$ Win $=8192$ Len $=0$ MSS=1460 WS=256 SACK_PERV $=1$ & \\
\hline & 30.64330700192 .168 .1 .10 & 192.168 .1 .20 & $\mathrm{TCP}$ & $66 \mathrm{http}>\mathrm{C3}$ [SYN, ACK] Seq=0 ACK=1 Win=8192 Len=0 MSS=1460 WS=256 SACK.PERV=1 & \\
\hline & 40.64384500192 .168 .1 .20 & 192.168 .1 .10 & & $54 \mathrm{C3}>$ http $[A C K]$ Seq $=1$ ACK $=1$ win $=65536$ Len $=0$ & \\
\hline & 50.64802400192 .168 .1 .20 & 192.168.1.10 & & 440 GET / server/Posthome, php HTTP/1.1 & \\
\hline & 60.76020000192 .168 .1 .10 & 192.168 .1 .20 & & 1514 [TCP segment of a reassembled PDU] & \\
\hline & 70.76131900192 .168 .1 .10 & 192.168 .1 .20 & & $596 \mathrm{HTTP} / 1.1200 \mathrm{OK} \quad($ text $/ \mathrm{html})$ & \\
\hline & 80.76182700192 .168 .1 .20 & 192.168 .1 .10 & & $54 \mathrm{C3}>$ http $[$ ACK $]$ Seq $=387$ ACK $=2003$ win $=65536$ Len $=0$ & \\
\hline & 90.80236100192 .168 .1 .20 & 192.168.1.10 & TCP & 54 C3 > http [FIN, ACK] Seq $=387$ ACK=2003 win $=65536$ Len $=0$ & \\
\hline & 10 0.82675600 Honeywel_09:15:07 & Broadcast & ARP & 42 who has 93.93 .67 .140 ? Tell 192.168.1.20 & \\
\hline & 110.83320600192 .168 .1 .10 & 192.168 .1 .20 & $T C P$ & 60 http $>$ C3 [ACK] Seq=2003 ACK=388 win=65536 Len=0 & \\
\hline & 120.83416600192 .168 .1 .10 & 192.168 .1 .20 & TCP & 60 http >c3 [FIN, ACK] Seq=2003 ACK=388 win=65536 Len=0 & \\
\hline & 130.83492200192 .168 .1 .20 & 192.168 .1 .10 & TCP & $54 \mathrm{C3}>$ http $[$ ACK] Seq $=388$ Ack $=2004$ win $=65536$ Len $=0$ & \\
\hline & 141.39517800192 .168 .1 .30 & 52.0 .86 .213 & TCP & $6614121>$ http [SYN] Seq=0 Win=8192 Len=0 MSS=1464 W5=256 SACK_PERV=1 & \\
\hline & 151.82681300 Honeywel_09:15:07 & Broadcast & & 42 who has 93.93 .67 .140 ? Tell 192.168 .1 .20 & \\
\hline & 162.29927400192 .168 .1 .30 & 52.0 .86 .213 & TCP & $6614123>$ http 「sYN] Sea $=0$ Win $=8192$ Len=0 MSS=1464 W5=256 SACK_PERV=1 & \\
\hline
\end{tabular}

Figure 18: Server - Client Communication Interception

Once again, upon TCP stream following of this

\subsection{Plaintext Server-Client Communication}

The information obtained showed that the response was using the "GET" form submission method as indicated by the first line. Note that packet, the information shown in Figure 19 was obtained.

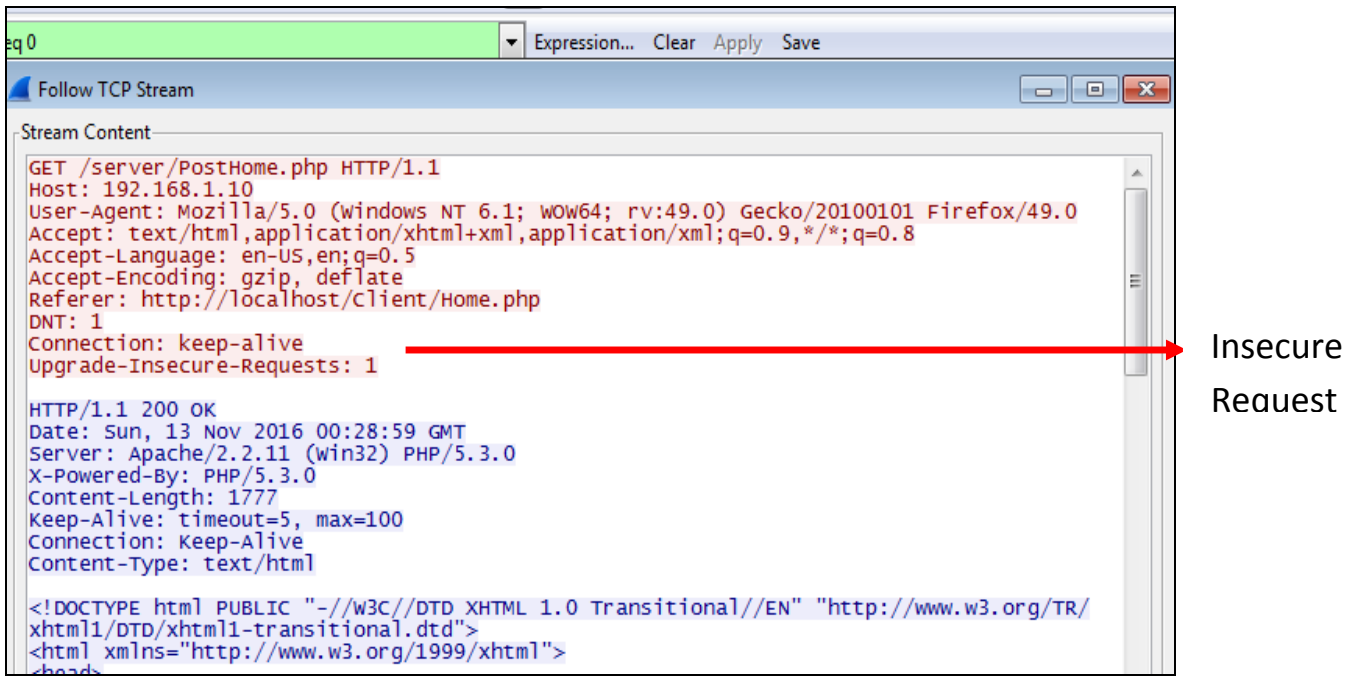

Figure 19: Plaintext Server-Client Communication 


\subsection{Scrambled Instant Message Packet}

The proposed port-based algorithm would scramble the packets such that the interception of this packet makes no sense to the intruder.
Figure 20 shows such kind of TCP packet scrambling in a typical instant message.

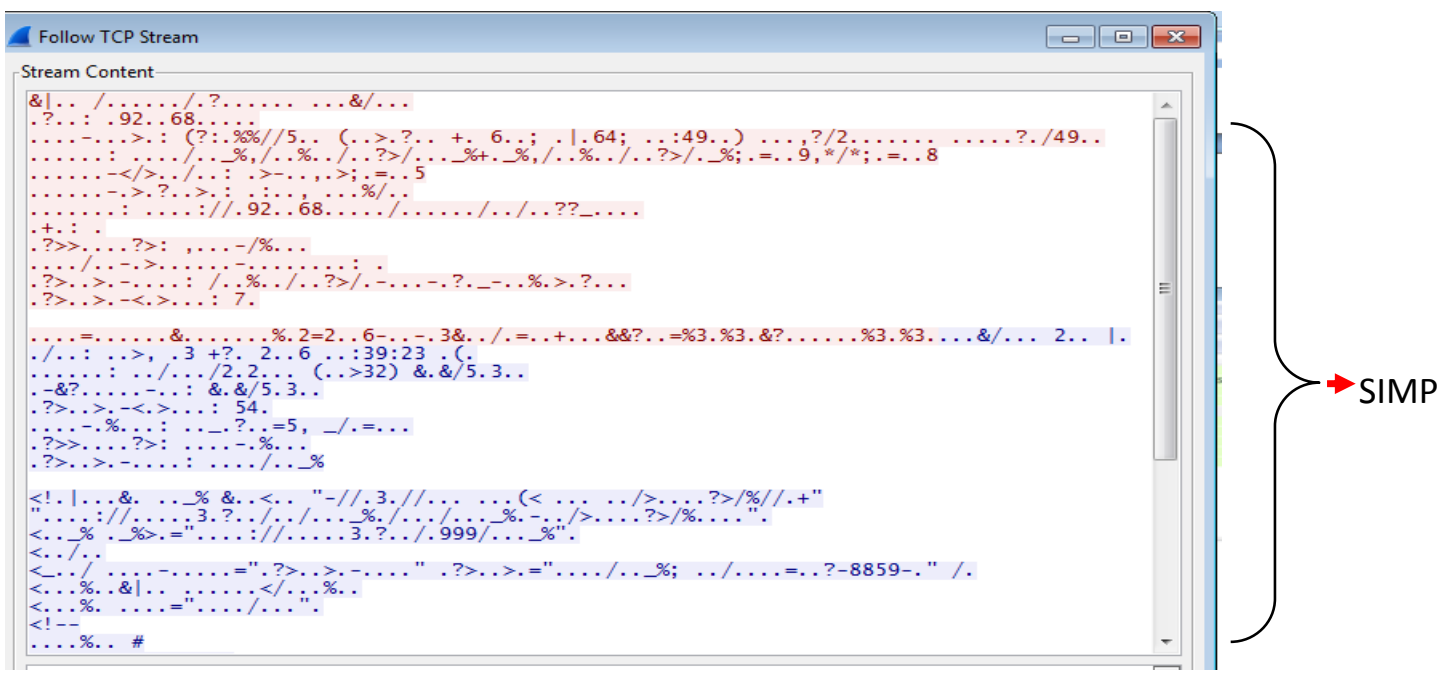

Figure 20: Typical Scrambled Instant Message Packet

This figure confirms the fact that if instant messages could be scrambled, then $t$ its content is meaningless to the human readers. In such a case, a decryption key will be required to turn this data into human readable format.

\section{CONCLUSIONS}

This paper sought to demonstrate the fact that instant messaging applications have numerous vulnerabilities, one of them being the transmission of messages in plaintext. To illustrate this, a prototype was developed in Java programming language using its networking sockets. The prototype was run in an Oracle VirtualBox VM environment. The results that were obtained demonstrate clearly that the instant messages are in plaintext in both the sending and receiving machines.

Moreover, data capture that was performed using Wireshak further reveals that the instant messages were not immune from interception and remote monitoring. Therefore, the researchers recommend secure protocols, strong authentication and message encryption at both the receiver and sender so that these messages are in human unreadable format in both terminals. In this way, eavesdropping and remote monitoring of the communication can be thwarted.

\section{REFERENCES}


1] Weissbrot \& Alison (2016). Car Service APIs Are Everywhere, But What's In It For Partner Apps? AdExchanger. ad exchanger.

2] Mark (2015). Private, Partner or Public: Which API Strategy Is Best For Business?. Programmable Web.

3] Z. Vinnie \& G. Belvin (2012). Silent circle instant messaging protocol.

4] S. Lin Z. Hao; X. Tao; L. Mingshu (2016). An Empirical Study on Evolution of API Documentation. International Conference on Fundamental Approaches to Software Engineering. Springer Berlin Heidelberg.

5] O. Wendell (2013). Cisco CCENT/ CCNA ICND1 100-101 Official Cert Guide. Pearson Education. pp. Ch. 1

6] M. Green (2014). Noodling about IM protocols.

7] Andreas \& Buchenscheit (2014). Privacy implications of presence sharing in mobile messaging applications. Proceedings of the 13th International Conference on Mobile and Ubiquitous Multimedia. ACM.

8] P. Jagwani (2016). Analyzing Instant Messaging Applications for Threats: WhatsApp Case Study. Department of Computer Science, Aryabhatta College, University of Delhi, Delhi (India).

9] T. Frosch C. Mainka, C. Bader, F. Bergsma, J. Schwenk, T. Holz (2016). How Secure is TextSecure. Ruhr University Bochum.

10] B. Smith (2013). UDP vs TCP security

11] G. Fahrnberger (2014). SIMS: A Comprehensive Approach for a Secure Instant Messaging Sifter. 